\title{
Wanderblöcke im Gebiet der Bergünerstöcke (Kt. Graubünden/Schweiz)
}

\section{Einleitung}

Nachdem TROLL im Jahre 1944 den Stand der Solifluktionsforschung ${ }^{1}$ weltweit zusammengefaßt hatte, wurde, auch in den Alpen, die beschreibende und genetische Grundlagenforschung intensiviert. Seit den siebziger Jahren werden elektronische Meßgeräte verwendet, welche die jährlichen Bewegungsbeträge der Formen aufzeichnen und damit Rückschlüsse auf frühere klimatische Verhältnisse erlauben.

Den Wanderblöcken wurde dabei kaum Beachtung geschenkt, obwohl sie bereits anfangs dieses Jahrhunderts von skandinavischen Forschern erstmals beschrieben worden waren. Doch weder TROLL (1944) noch, vorerst, FURRER (1954), der detaillierte Studien im Schweizerischen Nationalpark betrieb, führten sie in ihren Arbeiten auf.

Vermehrte Untersuchungen in den Alpen aber ergaben, daß die weitverbreiteten Wanderblöcke bei ihrer Genese Frostprozessen unterliegen. Wanderblöcke werden heute zur gebundenen ${ }^{2}$ Solifluktion gezählt. Dabei beschränkt sich die Untersuchung der Wanderblöcke mehrheitlich auf die Morphologie, während die Genese nur selten behandelt wird. Als Ausnahme sind die Studien von TUFNell $(1972,1976)$ und JAHN/CIELINSKA (1974) zu erwähnen. TUFNELLS Untersuchungen von 1965 bis 1975 konzentrierten sich jedoch fast ausschließlich auf das Gebiet von Moor House Reserve in Nordwestengland; daneben beschrieb er aber auch Wanderblöcke im Gebiet von Fiesch-Eggishorn im Kanton Wallis (Schweiz), dessen klimatische und topographische Verhältnisse einen Vergleich mit der Studienregion zuließen. Aus diesem Grund wurde die Klassifikation TUFNELls teilweise übernommen.

Im folgenden werden die Wanderblöcke im Raum Bergün-Savognin im Kanton Graubünden (Schweiz) detailliert beschrieben sowie Interpretationsversuche ihrer Genese unternommen.

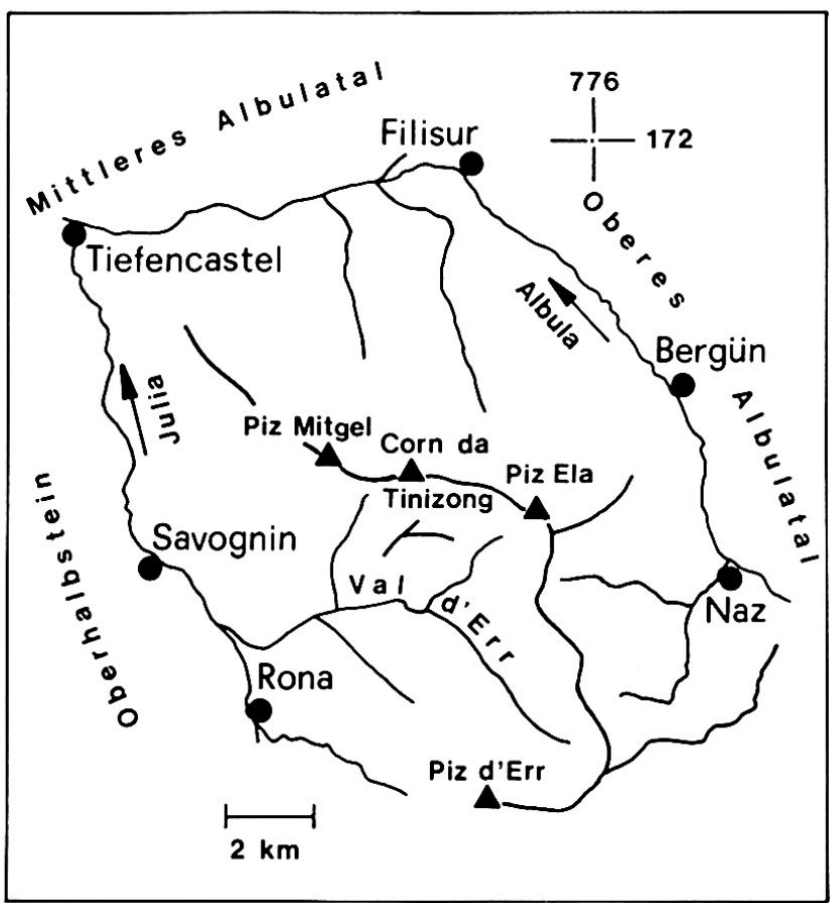

Fig. 1 Karte des Untersuchungsgebietes

\section{Arbeitsgebiet}

Das Untersuchungsgebiet liegt im Zentrum des Kantons Graubünden in den Schweizer Alpen. Im Osten wird es durch das Obere Albulatal begrenzt, im Norden durch das Mittlere Albulatal zwischen Filisur und Tiefencastel, im Westen durch die Julia und im Süden durch die Linie Rona-Piz d'Err-Naz (Fig. 1). Im Norden der Gipfelkette Piz Mitgel-Corn da Tinizong-Piz Ela verhindert das zumeist steile Gelände und das ungünstige Gestein (Hauptdolomit) die Entstehung von Formen, v.a. Wanderblöcke sind selten. Im Süden dieser Bergkette hingegen treten vegetationsbedeckte Hänge und damit auch Wanderblöcke häufiger auf, besonders im Val d'Err.

Werner Schwarz-Zanetti, dipl. Geograph, Geographisches Institut der Universität Zürich-Irchel, Winterthurerstr. 190, 8057 Zürich 


\section{Beschreibung von Wanderblöcken und deren näheren Umgebung}

\subsection{Allgemeines}

Diese Studie geht mit der Definition von HöLLERMANN (1964) einig, der Wanderblöcke als "größere Blöcke» bezeichnet, "die an der Stirnseite einen Stauchwulst, an der Rückseite eine Hohlform (Auswanderungsnische oder länger gestreckte Gleitbahn) aufweisen und sich in einem feinmaterialreichen Medium offensichtlich rascher als die Umgebung hangab bewegt haben» (Abb. 1).

Untersucht wurden 62 Wanderblöcke. Mit Funden zwischen 2175-2550 m ü. M. kommt die große Vertikaldistanz der Fundorte in den Bergünerstöcken nicht zum Ausdruck. Unter den nicht eindeutig als Wanderblöcke definierbaren Funden - sie sind nicht in der Fig. 2 aufgeführt - lag der tiefste bei $2060 \mathrm{~m}$ ü. M., der höchste auf $2640 \mathrm{~m}$ ü. M. Dazwischen ist die Verteilung außerordentlich regelmäßig, eine typische Höhenlage ist nicht erkennbar. Die steilen Wände der Bergünerstöcke verunmöglichen über $2600 \mathrm{~m}$ ü. M. das Auftreten von Wanderblöcken.

In der Literatur wird wiederholt auf die expositionsbedingte Abhängigkeit der Höhenlage hingewiesen. Die 62 gefundenen Blöcke rechtfertigen jedoch keine solche Untersuchung, zudem haben die Blöcke keine einheitliche Himmelsrichtung. Ähnliche Beobachtungen machte FURRER (1965), der in den Alpen 120 Wanderblöcke kartierte.

\subsection{Auswertung der Resultate}

Die Fig. 2 hält quantitative und qualitative Daten der 62 untersuchten Wanderblöcke fest. Die Ordinate gibt immer die relative Häufigkeit in Prozenten wieder, wogegen die Abszissenanschrift jeweils ändert.

\subsubsection{Gesteinsart}

Zwei Gesteinsarten dominieren im Untersuchungsgebiet: Granit und Dolomit. Der Anteil an Blöcken aus Kalk, Serizit, Diorit und Radiolarit ist bedeutend kleiner. Angaben über die Gesteinsart von Wanderblöcken sind in der Literatur selten, doch fand z. B. BROSCHE (1978) unter anderem auch mürbe, feine Sandsteine, Grauwacken und grobkörnige Sandsteine mit Mergelzwischenlagen.

Die Resultate aller Untersuchungen ergaben, daß alle hinreichend verwitterungsresistenten Gesteine Wanderblöcke bilden.

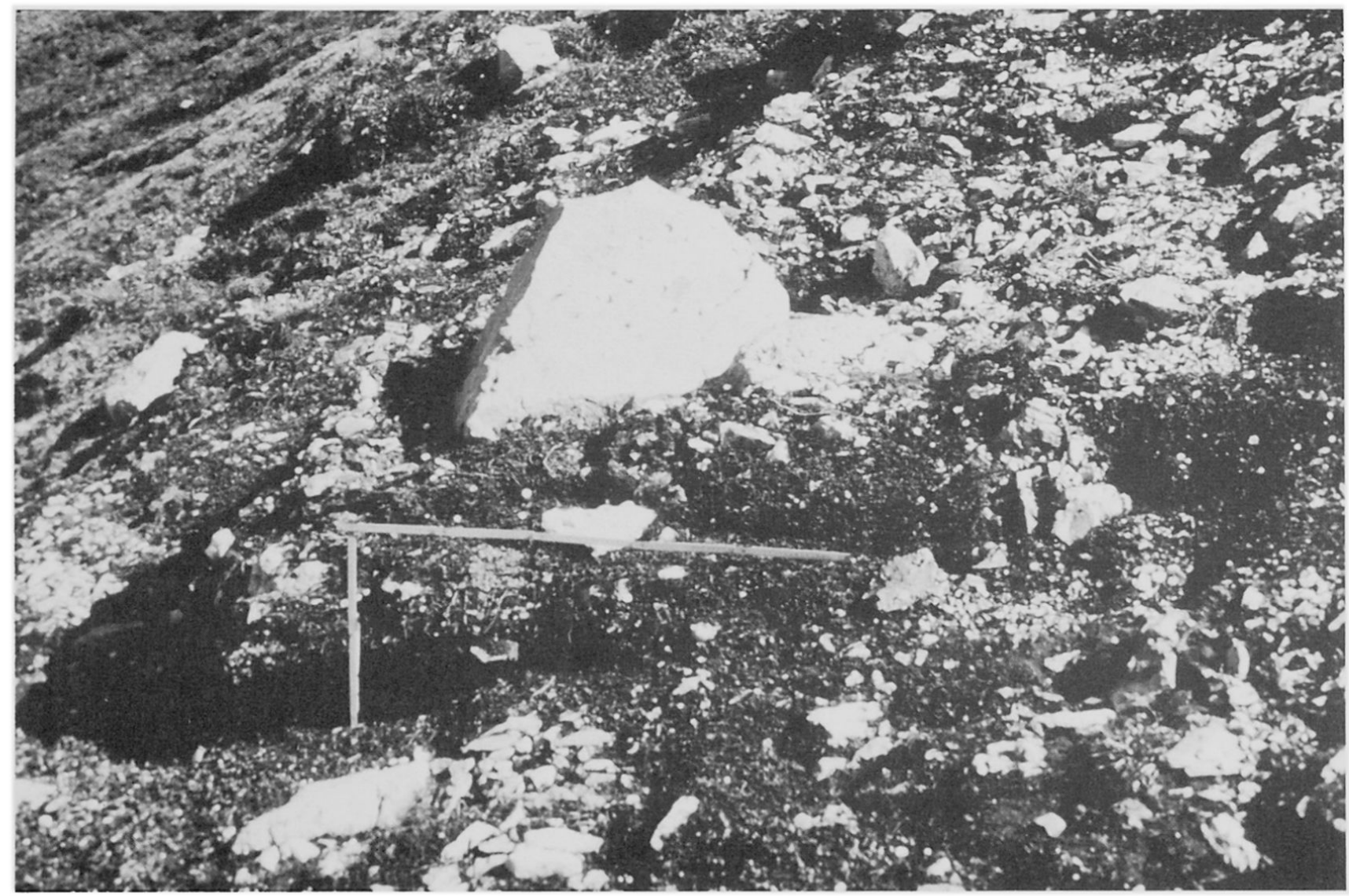

Abb. 1 Wanderblock mit starkem Wulst aus dem Gebiet von Andeer. Fließrichtung von rechts oben nach links unten. Photo: U. Brunner (Klappmeter: 100/40 cm) 


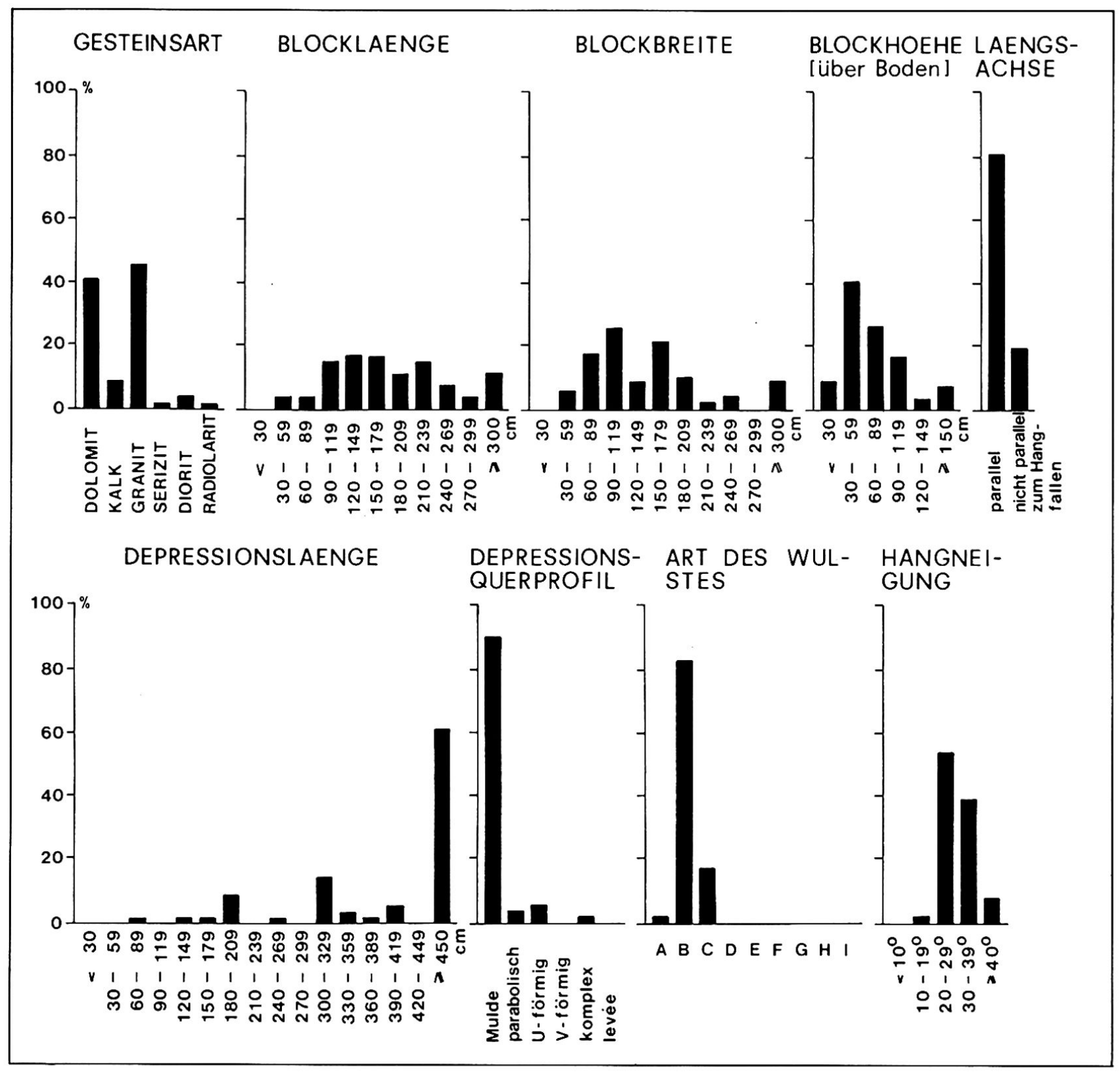

Fig. 2 Untersuchungsergebnisse

\subsubsection{Blocklänge, Blockbreite, Blockhöhe (über Boden) ${ }^{3}$}

Die Dimensionen eines Wanderblockes dürften bei seiner (möglichen) Bewegung wesentlich sein. Die Blocklängen verteilen sich gleichmäßig von 90 bis $300 \mathrm{~cm}$, solche unter $90 \mathrm{~cm}$ sind kaum vorhanden. Blockbreiten erreichen ihr Optimum zwischen 30 und $240 \mathrm{~cm}$. Die Verteilung der sichtbaren Blockhöhen ist uneinheitlich, dennoch treten sie vorwiegend zwischen 30 und $120 \mathrm{~cm}$ auf. Für die Volumenberechnung, die zusammen mit der Depressionslänge evtl. Rückschlüsse auf Geschwindigkeit und Hangabwärtsbewegung und Entstehungszeit erlauben würde, fehlt das Maß der durchschnittlichen Gesamtblockhöhe.

Welchen Einfluß die Form der Blöcke auf die Wanderbewegung hat, ist nicht geklärt; im Gebiet der Bergünerstöcke treten flache längliche und kurze hohe Blöcke genauso häufig auf wie rundliche oder eckige.

\subsubsection{Längsachse ${ }^{4}$}

Die Längsachse verläuft vorwiegend parallel zum Hangfallen. Mit der Ausrichtung der Längsachse in die Fallrichtung ist die Reibung wesentlich kleiner, als wenn die Längsachse schräg zum Hang verläuft. Liegt ausnahmsweise eine andere Ausrichtung der Längsachse vor, so könnte dies in der Topographie des Geländes begründet sein. Es kommt aber auch vor, daß sie gegenüber dem Hang leicht angehoben ist, d.h. daß sie schwächer geneigt ist als der Hang. Möglicherweise handelt es sich hierbei um fossile Blöcke, die talwärts auf den Stauchwulst aufgefahren sind. 


\subsubsection{Depressionslänge}

Bei der Depression kann eine Auswanderungsnische oder eine Schleifspur unterschieden werden, wobei auch eine Kombination möglich ist. Eine Schleifspur ist eine hinter einem hangabwärts rutschenden Block zurückgelassene Spur, die gegenüber der Umgebung etwas vertieft ist. Eine Auswanderungsnische ist der Ort, an dem ein Gesteinsblock lag, bevor er sich durch äußere Einflüsse (von neuem?) hangabwärts zu bewegen begann. In den Bergünerstöcken weisen zwei Drittel aller Blöcke Depressionslängen von über $450 \mathrm{~cm}$ auf. Im Gebiet von Fiesch-Eggishorn liegt das Schwergewicht einerseits zwischen 30 und $420 \mathrm{~cm}$ und andererseits über $450 \mathrm{~cm}$. Obwohl also in den Schweizer Alpen kein einheitliches Bild von Depressionslängen vorliegt, darf vom Überwiegen der Längenklasse über $450 \mathrm{~cm}$ gesprochen werden. Während die Auswanderungsnischen alle mehr oder weniger die gleiche halbmondförmige Form aufweisen, sind bei den Schleifspuren vier Varianten zu unterscheiden (Fig. 3). Nur wenige Wanderblöcke weisen keine gerade Schleifspur auf, Unebenheiten eines Hanges, aber auch Hindernisse wie Bäume oder große Felsbrocken können zu Richtungsänderungen des hangabwärts wandernden Blockes führen. Die Schleifspuren haben in den Bergünerstöcken eine maximale Länge von $25 \mathrm{~m}$, eine minimale von $2 \mathrm{~m}$. Es handelt sich hier aber immer um Minimalwerte, können doch andere Hangprozesse zur Zerstörung derselben füh-

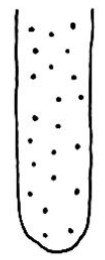

A

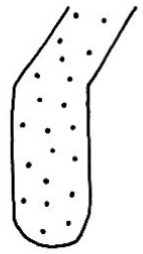

B

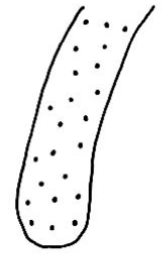

C

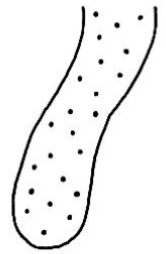

D
Fig. 3 Arten von Schleifspuren
(A) gerade
(B) eckig
(D) gewunden
(C) gekrümmt

ren. Die Depressionen hinter den Wanderblöcken weisen rein äußerlich beträchtliche Unterschiede auf (Fig. 4). Mit Abstand am häufigsten tritt Typ 1 auf, bei dem wohl eine Schleifspur, aber keine Auswanderungsnische $\mathrm{zu}$ sehen ist. Am zweithäufigsten tritt Typ 2 auf, wobei das Gesteinsmaterial unmittelbar hinter dem Block variiert. Nur vereinzelt wurden die Typen 3-8 gefunden.

\subsubsection{Depressionsquerprofil}

Nicht nur die Länge einer Depression, sondern auch ihr Querprofil kann über die Bewegung des Blockes Aufschluß geben. In Fig. 5 sind die sechs aufgenommenen Querprofile dargestellt. Die Zuordnung zu

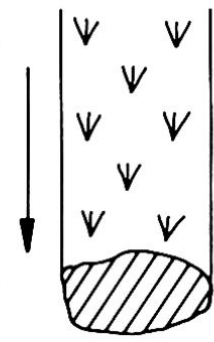

1

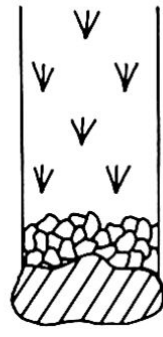

2

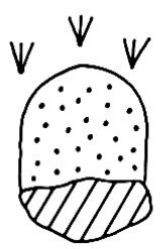

3

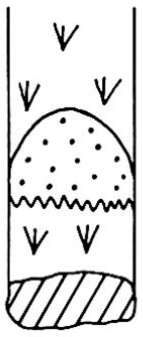

4

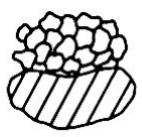

5

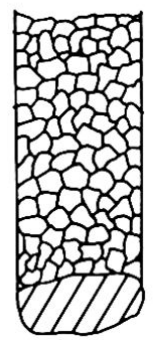

6

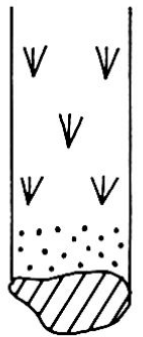

7

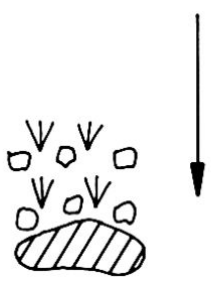

8

\section{QDD Wanderblock \\ $\therefore \therefore$ Auswanderungsnische \\ $|\vee|$ krautige Vegetation Steine $\because \because \because$ Feinerde}

Fig. 4 Depression von Wanderblöcken

(1) Schleifspur, die bis an den Block mit Vegetation bedeckt ist; keine Auswanderungsnische

(2) Schleifspur, die nicht bis an den Block mit Vegetation bedeckt ist, hinter dem Block grobes Gesteinsmaterial

(3) Auswanderungsnische und Schleifspur, ohne Vegetation

(4) Schleifspur mit Vegetation, Auswanderungsnische ohne Vegetation

(5) Keine Schleifspur ersichtlich, hinter dem Block grobes Gesteinsmaterial, das evtl. eine ehemalige Auswanderungsnische bedeckt.

(6) Schleifspur mit grobem Gesteinsmaterial gefüllt

(7) Schleifspur, die nicht bis an den Block mit Vegetation bedeckt ist, hinter dem Block keine Vegetation

(8) Depression mit Vegetation und Schuttmaterial 
einem dieser Profile fällt oft schwer, treten doch zahlreiche Zwischenformen auf. In den Bergünerstökken überwiegt die Muldenform deutlich die parabolische, die U-förmige und die komplexe Form; Profile mit V-Form und mit Seitenerhebungen wurden nie gefunden. Vergleichbare Untersuchungen in den Gebieten des Albulapasses sowie Grevasalvas im Oberengadin bestätigen diese Ergebnisse. Studien von TUFNELL (1972) im Gebiete von Fiesch-Eggishorn ergaben demgegenüber mehr parabolische Profile als Muldenformen. Fast immer entsprach die Breite der Querprofile der Breite des Blockes. Es kam auch vor, daß das Querprofil breiter oder aber schmaler war als der Block. Im ersten Fall könnten Erosionsprozesse vorliegen; denkbar ist auch, daß die Längsachse eines Blockes beim Rutschen hangabwärts nicht immer parallel zum Hang verlaufen ist und erst in der Nähe des heutigen Lageortes die Längsachse parallel zum Hang ausgerichtet hat. Im zweiten Fall dürften Hangprozesse im Spiel sein, welche das Profil nachträglich verengten. Bei einem Wanderblock konnte beobachtet werden, daß die Schleifspur ihr Querprofil verändert hatte. Die Schleifspur hat eine Länge von $13 \mathrm{~m}$, im obersten Teil V-Form, darauf U-Form und etwa $6 \mathrm{~m}$ über dem Block wechselt die U-zur Muldenform. Möglicherweise hat eine intensive Bewegung des Hanges diese Verengung der Schleifspur bewirkt. Die Feldbeobachtung läßt jedoch in diesem Fall eher auf eine Drehung des Blockes während der Rutschphase schließen.

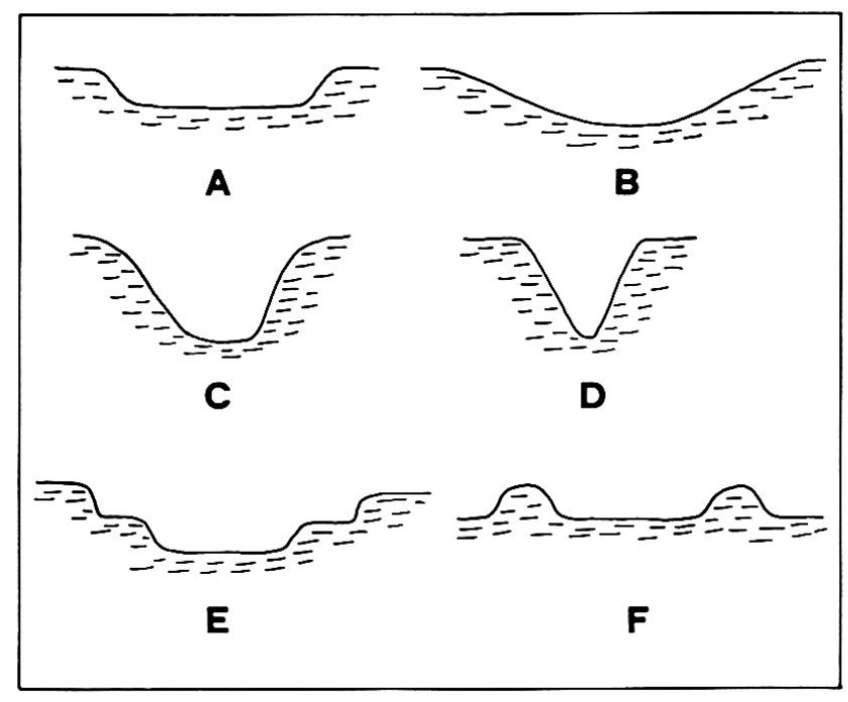

Fig. 5 Querprofile von Depressionen (nach TUFNELL 1972)
(A) muldenförmig
(B) parabolisch
(D) V-förmig
(C) U-förmig
(F) mit Seitenerhebungen

\subsubsection{Art des Wulstes}

TUFNELL (1972) unterschied sechs einfache sowie drei Doppelwülste. Dieses Klassifikationssystem konnte aufgrund der Felderfahrung übernommen werden, neue Wulstarten mußten nicht mehr dazugenommen werden (Fig. 6). In den Bergünerstöcken dominiert

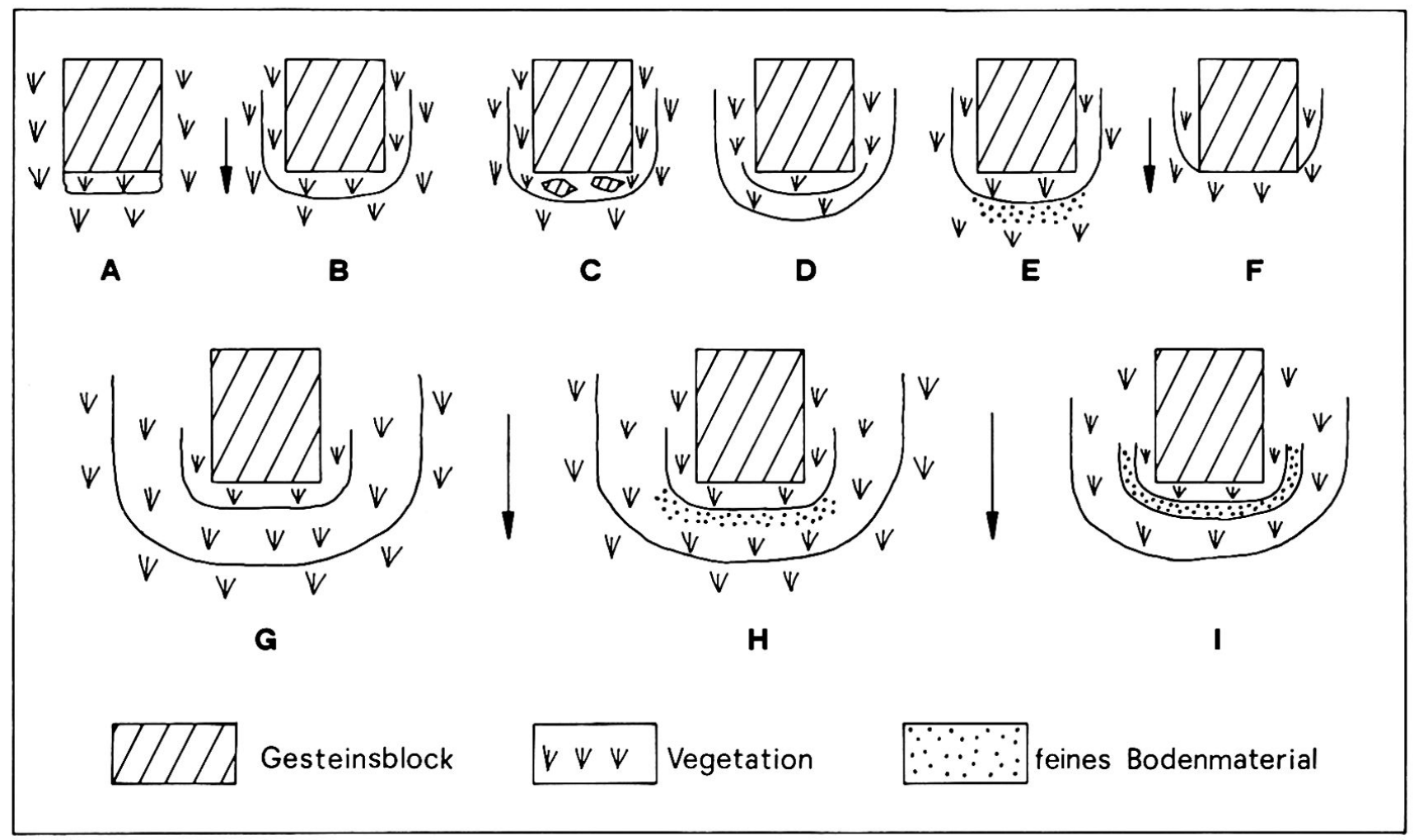

Fig. 6 Aufsicht von Wulstarten (nach TUFNELL 1972)

A-F Einfache Wulste G-I Doppelwulste 
Typ B: vor und neben dem Block entsteht ein durch den hangabwärts bewegten Block zusammengeschobener Gürtel von Bodenmaterial. Von gewisser quantitativer Bedeutung ist auch noch Typ C. Im Unterschied zu B schiebt dieser kleine Gesteinsstücke vor sich her, die im Wulst deutlich sichtbar sind. Die Wülste können wohl morphologisch exakt aufgenommen werden, doch ist deren Funktion während und nach ihrer Bildung praktisch unbekannt. Es scheint, da $ß$ hangabwärts bewegte Blöcke durch die Volumenzunahme der sich bildenden Wülste gebremst und zum Stillstand gebracht werden. Ob zu diesem Zeitpunkt die Bewegung des Blockes für immer abgeschlossen wird, oder ob später eine neue Bewegungsphase beginnt, könnte von der Größe des Wulstes und auch von dessen Vegetationsbedeckung im Verhältnis zum Druck des Blockes ${ }^{5}$ abhängen. Praktisch alle Wülste in den untersuchten Gebieten der Bündner Alpen waren mit alpinem Rasen bedeckt. $\mathrm{Da}$ sich aber dennoch Blöcke mit gut ausgebildetem, vegetationsbedecktem Wulst noch bewegen können, soll in Kap. 5 gezeigt werden.

\subsubsection{Hangneigung}

Grundsätzlich kommen Wanderblöcke nur auf stark geneigten Hängen vor. Im Raume der Bergünerstöcke überwiegen Neigungswinkel von $20-40 \mathrm{Grad}$; diese Resultate unterscheiden sich nur unwesentlich von denen anderer Autoren, die im Alpengebiet gearbeitet haben.

Der Versuch, einen Zusammenhang zwischen der Depressionslänge und der Hangneigung zu finden, führte zu keinen signifikanten Ergebnissen.

\section{Genese}

\subsection{Herkunft der Blöcke}

TUFNELL (1976) schreibt, daß viele Blöcke durch Frostsprengung von anstehendem Gestein gelöst werden, andere dürften glazialen Ursprungs sein, also Erratiker. Im Gebiet der Bergünerstöcke handelt es sich ausschließlich um durch Frostsprengung entstandene Blöcke; ein Vergleich mit der Gesteinsart des Blockes und den hangaufwärts vorkommenden anstehenden Gesteinen beweist dies.

Möglicherweise fallen die Blöcke in den Schnee und sinken im Frühjahr während des Abschmelzens der Schneedecke auf den Hang. Nach TUFNELl (schriftl. Mittlg. 1982) gibt es zwei weitere Möglichkeiten:

- Die Blöcke fallen auf stark durchnäßten Boden, oder

- sie lösen sich von anstehendem Fels nahe der Bodenoberfläche und bleiben dort so lange liegen, bis Solifluktionskräfte zu wirken beginnen.

Ob bei diesen drei Fällen die Hangabwärtsbewegung des Blockes unmittelbar nach dem Auftreffen auf den Hang beginnt oder ob zuerst eine Ruhephase eintritt,

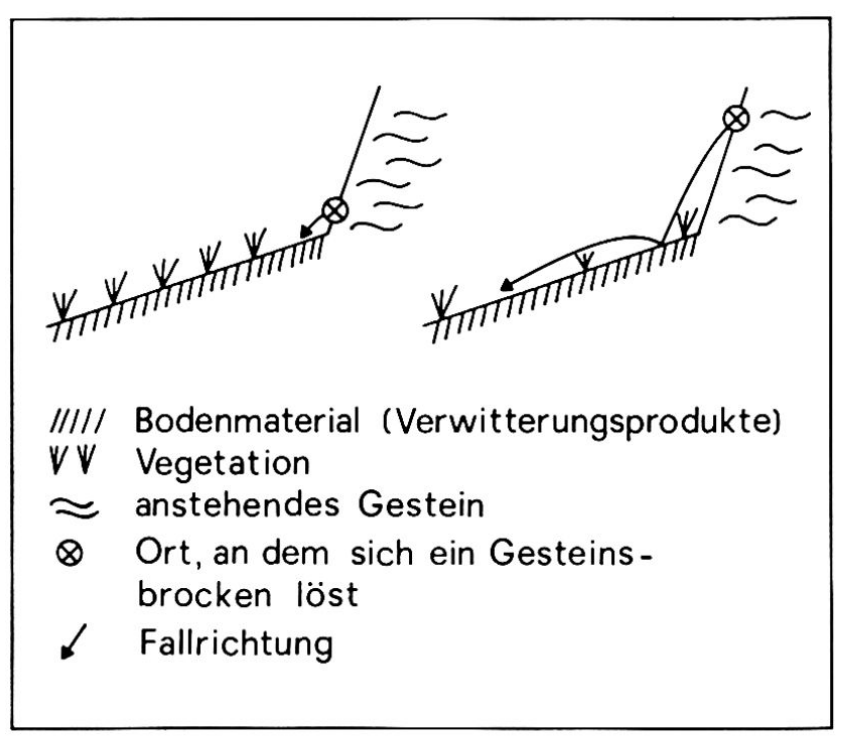

Fig. 7 Weg des anstehenden Gesteins auf den Hang

kann heute noch nicht beantwortet werden. Bis jetzt ging diese Studie davon aus, daß die auf den Hang fallenden Steine in der Nähe des Anstehenden zum Stillstand gelangt sind (Fig. 7, links). Es gibt aber auch Blöcke, die in großer Distanz vom Anstehenden vorerst zum Stillstand gekommen sind (Fig. 7, rechts), und erst danach ihre Wanderung begannen, was durch eine kurze Depression bewiesen ist.

Ursache dieser großen Entfernung vom Ursprungsort dürfte die große vertikale Fallstrecke des losgelösten Gesteins sein. Die Geschwindigkeit des auf den Hang auftreffenden Blockes ist dann so gro $\beta$, da $\beta$ eine weitere Bewegung hangabwärts erfolgt. Eine Hangverflachung oder Hindernisse beenden dann die Bewegung des Blockes ${ }^{6}$.

\subsection{Ursachen der Blockbewegung}

SCHMID (1958) und TUFNELL (1976) sehen in der Frosthebung eine Möglichkeit, die Blockbewegung zu initiieren. Daneben gibt TUFNELL aber noch drei weitere Erklärungsvorschläge:

- Gestein hat die Fähigkeit, Wärme zu speichern und zu leiten: Es ist vielfach früher schneefrei als die vegetationsbedeckte Umgebung, so daß Schmelzwasser über die Oberfläche des Gesteins fließt und vor dem Block eine größere schneefreie Zone schafft. Damit entsteht unmittelbar vor dem Block eine Stelle, die Frostprozessen länger ausgesetzt ist und die Wanderung des Blockes erleichtert.

- Es herrscht ein spezielles Feuchtigkeitsangebot hinter dem Stein während mehreren Monaten im Jahr: Schneeflecken können je nach Topographie des Geländes bis weit in den Frühling hinter einem Block liegen bleiben und hinter diesem ein ständiges Reservoir von Wasser bilden, das in den noch kalten Frühlingsmonaten gefriert und den Block hangabwärts drückt. 
- Die Bewegungen erfolgen z.T. auf einer gefrorenen Unterlage: Die oberen Bodenschichten tauen auf und bilden eine "Schmierseife» auf der gefrorenen Unterlage.

Diese Bewegungsmöglichkeiten sind aber immer abhängig von geologischer Position, Höhenlage, Exposition, Hangneigung, Durchfeuchtungsverhältnissen, Schneedeckendauer und -mächtigkeit (FRITZ 1982, schriftl. Mittlg.). DORIGO (1969) kam zu ähnlichen Resultaten, wobei er die Untersuchung der Reibungsverhältnisse ${ }^{7}$ zwischen Block und Unterlage als entscheidenden Ansatz für die Erscheinung von Wanderblöcken wertet. Im Gebiet der Bergünerstöcke treten nur zwei Wanderblöcke in Erscheinung, die in jüngerer Zeit aus ihrer ursprünglichen Stellung herausgelöst wurden und sich hangabwärts bewegten. Daraus läßt sich am besten eine mögliche Rekonstruktion des Bewegungsvorganges und deren Ursache ersehen, was in Kap. 5 beschrieben werden soll.

\subsection{Bewegungsmessungen}

JAHN und CIELINSKa haben in den Sudeten Polens die Bewegungen von vier Blöcken zwischen 1960 und 1970 aufgezeichnet. Bei der Untersuchung ging man von der Vermutung aus, daß die Bewegung der Blöcke von der Korngrößenzusammensetzung des Bodens und seinen physikalischen Eigenschaften, der Vegetation, dem Klima und v. a. der Hangneigung abhängt. Die Untersuchung ergab aber keine Abhängigkeit zwischen Bewegungsbetrag und verschiedenen Hangneigungen. Ebenfalls eine Meßreihe über 10 Jahre (1965-1975) führte TUFNELL $(1972,1976)$ in Moor House Reserve durch. Er untersuchte dabei fünf Blöcke an Hängen mit verschiedenen Neigungswinkeln $(5,7,21,22 \mathrm{Grad})$. Die jährlichen Bewegungsbeträge der einzelnen Blöcke schwanken zwischen 0 und $8 \mathrm{~cm}$. Die Jahresbeträge eines einzelnen Blockes können von 1965 bis 1975 stark differieren. Für die Periode 1967 wurden Messungen zur Feststellung von Schwankungen innerhalb des gleichen Jahres unternommen. TUFNELL (1976) stellte dabei fest, daß die Bewegung in den kälteren Monaten des Jahres größer ist als während des Sommers.

Diese Bewegungsbeträge stehen im Gegensatz zu Beobachtungen in den Alpen, fand doch FURRER (1965) «Wanderblöcke» mit einer «frischen», vegetationsfreien Schleifspur von mehreren m Länge (bis über $10 \mathrm{~m}$ )». Solche Erscheinungen konnten im Gebiet der Bergünerstöcke nicht beobachtet werden $60 \%$ aller Schleifspuren waren über $450 \mathrm{~cm}$ lang, wobei die Rutschungen in einem Zug erfolgt sein dürften. Es scheint, daß die Blöcke von Zeit zu Zeit hangabwärts rutschen und zum Stillstand gelangen, wenn die Vegetation vor dem Block die Bewegung stark hemmt. Die Vermutung, daß Blöcke periodisch eine bestimmte Strecke rutschen, kann bis heute nicht generell gelten. Für einen Wanderblock im hinteren Val d'Err, auf einer Höhe von $2380 \mathrm{~m}$ ü. M., südwest- exponiert, auf einem Hang mit einer Neigung von über $40 \mathrm{Grad}$, trifft dies aber mit ziemlicher Sicherheit zu. Der auf einer Moräne liegende Block ist $220 \mathrm{~cm}$ lang, $230 \mathrm{~cm}$ breit und hat eine Höhe von $75 \mathrm{~cm}$ über Boden. Der Wulst ist schön ausgebildet, die Schleifspur mißt über $20 \mathrm{~m}$. Die Depression kann in eine vegetationslose Auswanderungsnische und in eine vegetationsbedeckte Schleifspur unterteilt werden. Gesamthaft wurden bei diesem Block an 12 Stellen Bodenproben entnommen, 3 Bohrstellen befinden sich in der Auswanderungsnische, 4 in der Schleifspur, 2 neben der Schleifspur und 3 vor dem Wulst. Die Profilskizzen innerhalb und außerhalb der Schleifspur, auch im Vergleich miteinander, weisen in ihren Horizontmächtigkeiten keine wesentlichen Unterschiede auf. Auch der Wassergehalt der einzelnen Bodenproben ist fast identisch. Eine Ausnahme bilden die Proben in der vegetationslosen Auswanderungsnische. Die Bedingungen bezüglich des Wasserhaushaltes (z.B. Verdunstung) haben sich somit an dieser Stelle verändert. Unterschiede zwischen Schleifspur und Umgebung sind aber den $\mathrm{pH}$-Berechnungen zu entnehmen. Außerhalb der Schleifspur erfolgte eine stärkere Versauerung als innerhalb. Es scheint, als ob innerhalb der Schleifspur die Verwitterung noch nicht sehr lange ausgesetzt habe. Welche Prozesse für diese unterschiedliche Bodenentwicklung ausschlaggebend sind, kann an dieser Stelle nicht beantwortet werden; möglicherweise spielt das Hangwasser eine entscheidende Rolle. Aufgrund der Profilaufnahmen sowie der $\mathrm{pH}-$ Werte innerhalb der Schleifspur darf aber angenommen werden, daß dieser speziell untersuchte Wanderblock ohne Unterbrechung über $20 \mathrm{~m}$ gerutscht ist.

\subsection{Bedeutung des Wulstes}

Blöcke mit kräftig ausgebildeten Wulsten, in Abhängigkeit von Hangneigung und Blockgröße, sind vermutlich fossil. Blöcke mit kleinen Wulsten können aber, wie das Beispiel aus dem Val d'Err (Kap. 5) zeigt, diesen bei starker Durchfeuchtung durchbrechen und so lange hangabwärts rutschen, bis eine verstärkte Wulstbildung die Fahrt hemmt. Es scheint nun aber, daß sich auch "fossile» Blöcke noch bewegen können. Die ehemals meist parallel zum Hang gerichtete längste Achse eines Wanderblockes wurde durch die bereits beschriebenen Prozesse (v.a. Frosthebung) leicht angehoben. Er wird möglicherweise auf den Wulst aufgeschoben, was auch die schon erwähnten Doppelwulste erklären könnte. Eine Beobachtung, die diese Vermutung unterstützt, machte BRUNNER (1977) im Raume Grevasalvas, als er nach wolkenbruchartigen Niederschlägen reaktivierte Wanderblöcke fand. Interessant ist, daß sich diese Blöcke aufstellten, d. h. ihre Hauptebene zum Hangwinkel veränderten, aber nicht hangabwärts rutschten; wahrscheinlich hat hier der kräftige Wulst als nicht zu überwindendes Hindernis gewirkt. 


\section{Beispiel eines Wanderblockes im Val d'Err}

Der Wanderblock aus dem Val d'Err ist $130 \mathrm{~cm}$ lang, $100 \mathrm{~cm}$ breit und $60 \mathrm{~cm}$ über Boden. Der Block wurde erstmals im Sommer 1980 kartiert. Hinter dem Block befand sich eine ca. $5 \mathrm{~m}$ lange, gerade, vegetationsbedeckte Schleifspur, und um den Block zog sich ein Wulst. Eine andere Situation präsentierte sich am 30. August 1981: der Block (Abb.2) hatte sich aus der ursprünglichen Verankerung gelöst und sich ungefähr $2 \mathrm{~m}$ hangabwärts verschoben. Mit Blick von oben zeigt sich das in Fig. 8 gezeichnete Bild. Aufgrund der vegetationslosen Auswanderungsnische und des zerstörten Wulstes kann noch nicht eindeutig auf eine erst vor kurzem erfolgte Bewegung geschlossen werden. Die vegetationsbedeckte Strecke zwischen der Auswanderungsnische und dem Hinterende des Blockes ist aber ein eindeutiger Beweis, denn sie ist mit feiner Erde bedeckt, welche bei Niederschlägen ohne Zweifel bald weggeschwemmt worden wäre. $\mathrm{Da}$ die Vegetation erst nach der Blockbewegung mit nachgeschwemmter Feinerde aus der Auswanderungsnische bedeckt worden ist, kann ausgeschlossen werden, da auf dem Feinmaterial deutliche Rutschspuren zu erkennen sind. Einen ersten Anhaltspunkt zur erneuten Aktivierung gibt die Auswanderungsnische; das Feinmaterial unter dem Stein eignet sich ausgezeichnet für Kammeisbildung, was zu einer Hebung des Blockes führen muß. Der Block ist oberflächlich abgerutscht, d.h. über die Vegetation hinweg. Ohne eine starke Herabsetzung der Reibung zwischen Block und Unterlage wäre dies aber kaum möglich gewesen. Vermutlich kam diese Reibungsverminderung durch eine starke Durchfeuchtung des Hanges zustande, fiel doch Ende Juli 1981 bis in tiefe Lagen nochmals über ein halber Meter Neuschnee, der in den darauffolgenden Wochen schnell schmolz. Möglicherweise lösten hangabwärts rutschende Schneemassen noch zusätzlich den Block aus seiner Verankerung. Mit einiger Sicherheit kann man also sagen, daß Frostprozesse im allgemeinen für die Bewegungsauslösung eine entscheidende Rolle spielen.

Am 9. September 1982 erfolgte eine Überprüfung der im Jahre 1981 vorgefundenen Situation. Überraschend hatte sich der Block nicht mehr weiterbewegt, sondern begann sich wieder einzutiefen. Das Feinmaterial zwischen Block und Auswanderungsnische war mehr oder weniger weggeschwemmt worden. Die Vermutung, daß sich Blöcke periodisch bewegen, dann aber um einen großen Betrag, dürfte somit bestärkt worden sein.

\section{Summary}

62 ploughing blocks (Wanderblöcke) were studied in the region of the Bergünerstöcke located in the eastern Swiss Alps by a classification system based on TUFNELL. The results obtained didn't differ signifi-

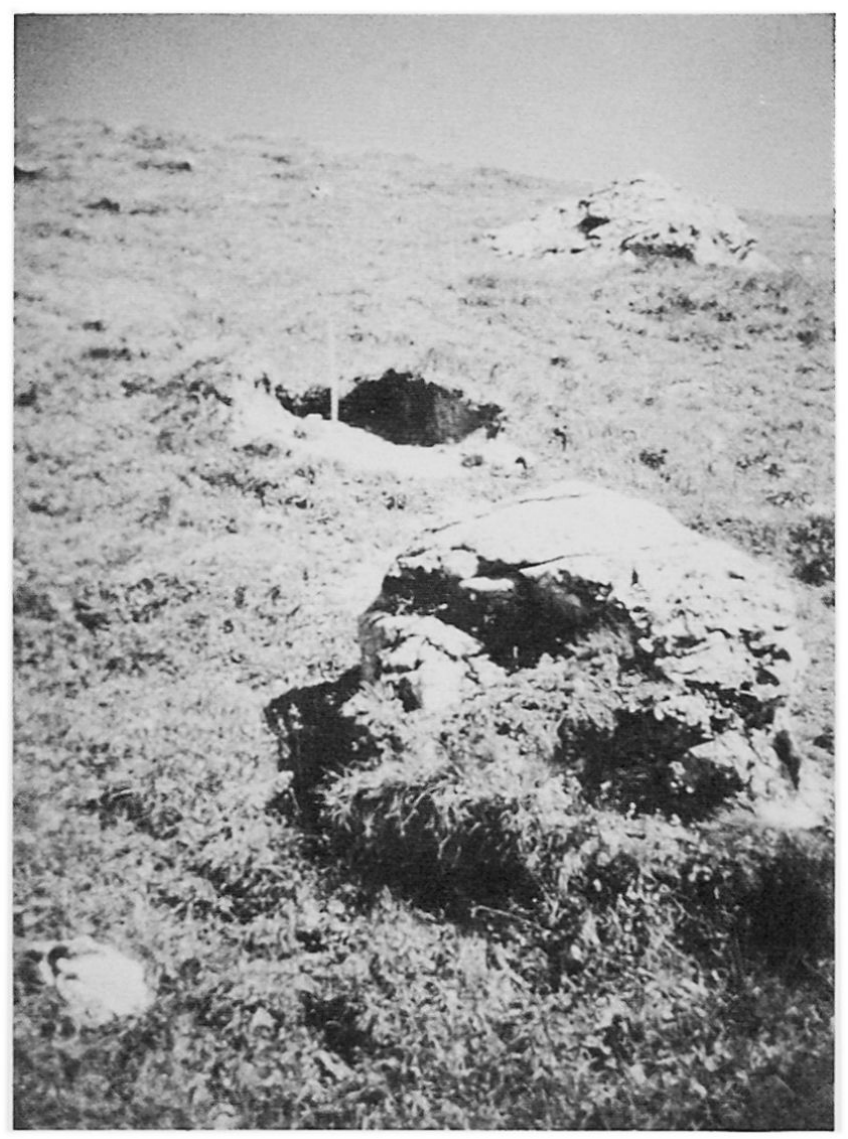

Abb. 2 Aus der Verankerung gelöster hangaufwärts betrachteter Wanderblock aus dem Val d'Err.

(2500 m ü. M., 774.525/158.675, Klappmeter: $60 \mathrm{~cm}$ )

cantly from former investigations in the Alps. It has been observed that lengths of more than 4.5 meters are dominating at the depressions and that they show a single slide over a large distance. The origin of blocks is attributed to frost breaking. The path on the slopes as well as the causes of the block moving are not clear yet. The initial cause of movement on the slope is predominantly attributed to frost processes where for example the unfrozen layer on top of the frozen layer represent an easy downsliding media. The length of depressions show that the movements take place periodically and over a quite large distance. Numerous soil samples have been taken in an elongate (Schleifspur) over a length of more than 20 meters in the rear Err Valley (Val d'Err) and it could be demonstrated that the block must have moved uninterruptedly. Recent movements were only found in two cases. It is known of a block in the rear Err Valley that it broke through the mound (Wulst) and slided down 2 meters on top of the vegetation. Due to the fine soil material in the niche-shape (Auswanderungsnische) susceptible to frost, the release by frost has been proved. When the movement has started, slope as well as friction play the most important role of the movement. Therefore it seems that blocks move periodically and then if at all over large distances. 


\section{Anmerkungen}

'Definition nach TROLL (1947, zit. in SCHENK 1953, S. 197): "Solifluktion im weitesten Sinne ist die Erscheinung, daß unter der Wirkung langdauernder jahreszeitlicher oder kurzdauernder, sich häufig wiederholender bis allnächtlicher Gefrornis des Bodens eine lebhafte Verlagerung der Bodenteilchen stattfindet, die sich auf ebenem Gelände in der Bildung von Bodenstrukturen oder Bodentexturen (Frostgefügeboden), auf ge-

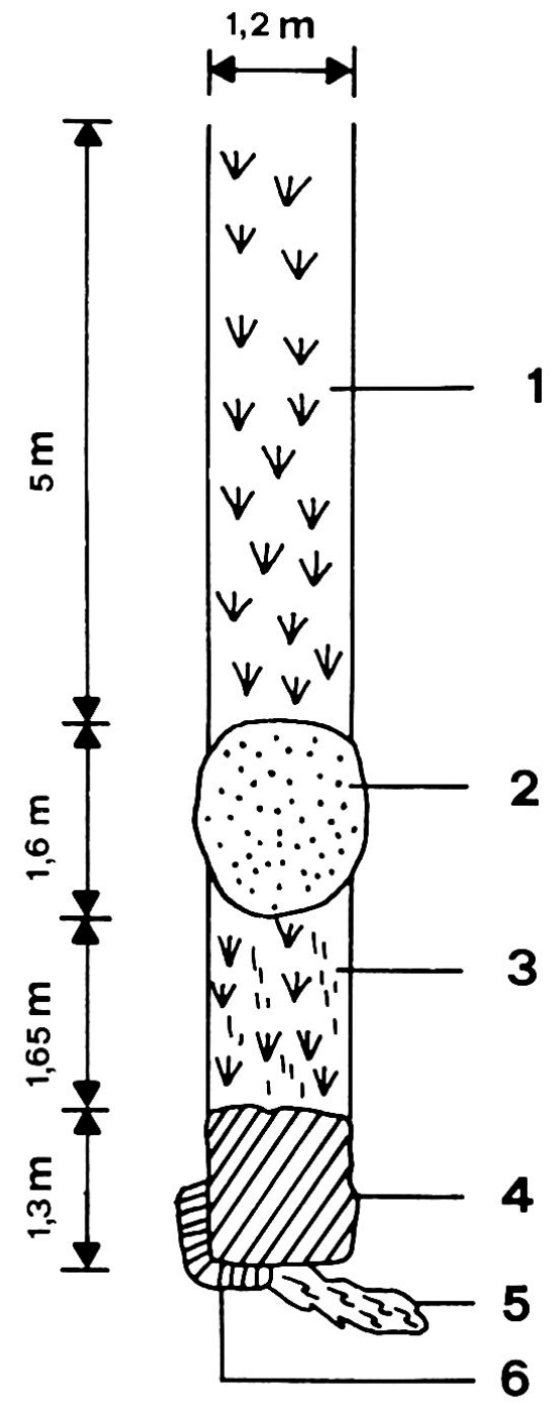

Fig. 8 Situation an einer Fundstelle im hinteren Val d'Err (774.150/158.875, $2380 \mathrm{~m}$ ü. M.)

(1) ehemalige Schleifspur, vegetationsbedeckt

(2) Auswanderungsnische mit feiner Erde, ohne Vegetation

(3) neue Schleifspur, die Vegetation wurde nicht zerstört, sie ist mit feiner Erde bedeckt

(4) Wanderblock

(5) aufgerissener Teil des Wulstes

(6) mehrheitlich kompakter Wulst neigtem Gelände auch bei ganz geringem Gefälle in einem beträchtlichen hangabwärts gerichteten Massentransport (Frostbodenversetzung) äußert.”

${ }^{2}$ Neben der gebundenen Solifluktion (auf geschlossener Vegetationsdecke) unterscheidet man noch die gehemmte (auf karger Vegetation) und die ungebundene (auf vegetationsfreien Flächen).

${ }^{3}$ Die Blocklänge wurde hangabwärts, die Blockbreite quer zum Hang gemessen.

${ }^{4}$ Unter der Längsachse ist die größte Länge eines Blockes zu verstehen; sie kann entweder parallel oder nicht parallel zur Fallrichtung verlaufen.

${ }^{5}$ Der Druck des Blockes auf den Wulst ist eine Funktion von Blockvolumen und -gewicht sowie der Hangneigung.

${ }^{6}$ Die kinetische Energie (Energie der Bewegung) ist in Fig. 7 (rechts) größer als links, nimmt sie doch linear mit der Geschwindigkeit im Quadrat zu $\mathrm{E}_{\mathrm{kin}}=\mathrm{mv}^{2} / 2$. Die Geschwindigkeit wiederum ist beim freien Fall von der durchfallenen Höhe abhänging $(v=\sqrt{2 g h})$.

${ }^{7}$ Die Reibung ist $u$. a. eine Funktion des Klimas (Niederschlag, Temperatur), der Vegetation und der Topographie.

\section{Literatur}

BROSCHE, K.-U. (1978): Beiträge zum rezenten und vorzeitlichen periglazialen Formenschatz auf der iberischen Halbinsel. In: Abh. des Geogr. Institutes der Freien Univ. Berlin. Sonderheft, Band 1.

BRUNNER, U. (1977): Solifluktions- und Vegetationsgrenzen im Raum Grevasalvas (Oberengadin). Diplomarbeit Geogr. Inst. Univ. Zürich.

DORIGO, G. (1969): Untersuchungen zur (gebundenen) Solifluktion am Sustenpaß und auf der Melchsee-Frutt. Diplomarbeit Geogr. Inst. Univ. Zürich.

FURRER, G. (1954): Solifluktionsformen im Schweizerischen Nationalpark. Diss. Univ. Zürich.

FURRER, G. (1965): Die Höhenlage von subnivalen Bodenformen untersucht in den Bündner und Walliser Alpen, verglichen mit den Verhältnissen im oberen Braldo- und Biafotal (Karakorum). Habil. Univ. Zürich.

HÖLLERMANN, P.W. (1964): Rezente Verwitterung, Abtragung und Formenschatz in den Zentralalpen am Beispiel des oberen Suldentales (Ortlergruppe). In: Zeitschrift für Geomorphologie Supplementband 4.

JAHN, A./CIELINSKA, M. (1974): The rate of soil movement in the Sudety Mountains. In: Abhandlung der Akademie der Wissenschaften in Göttingen, Mathematisch-Physikalische Klasse, III. Folge, Nr. 29.

SCHWARZ-ZANETTI, W. (1982): Solifluktion im Raum Bergün-Savognin, mit besonderer Berücksichtigung von Wanderblöcken. Diplomarbeit Geogr. Inst. Univ. Zürich.

TROLL, C. (1944): Strukturböden, Solifluktion und Frostklimate der Erde. In: Geolog. Rundschau, Band 34, Heft 7/8.

TUFNELL, L. (1972): Ploughing blocks with special reference to north-west England. In: Biuletyn Peryglacjalny, Nr. 21.

TUFNELL, L. (1976): Ploughing block movement on the moor near House Reserve (England), 1965-75. In: Biuletyn Peryglacjalny, Nr. 26. 